\title{
On symmetries and conserved quantities in Nambu mechanics
}

\author{
M. Fecko', a) \\ Department of Theoretical Physics, Comenius University, Bratislava, Slovakia
}

In Hamiltonian mechanics, a (continuous) symmetry leads to conserved quantity, which is a function on (extended) phase space. In Nambu mechanics, a straightforward consequence of symmetry is just a relative integral invariant, a differential form which only upon integration over a cycle provides a conserved real number. The origin of the difference may be traced back to a shift in degrees of relevant forms present in equations of motion, or, alternatively, to a corresponding shift in degrees of relevant objects in action integral for Nambu mechanics.

PACS numbers: 02.40.-k, 45.20.Jj, 47.10.Df, 11.30.-j, 45, 02.30.Hq

Keywords: Hamiltonian mechanics, Nambu mechanics, symmetry, conserved quantity, integral invariant

\section{INTRODUCTION}

According to seminal paper of Emmy Noether (Ref. 1, see also Ref. 2), there is a close correspondence between symmetries of action integral and conserved quantities for the dynamics given by the action.

Recall briefly, how it works in Hamiltonian mechanics. First, Hamilton equations

$$
\dot{q}^{a}=\frac{\partial H}{\partial p_{a}} \quad \dot{p}_{a}=-\frac{\partial H}{\partial q^{a}}
$$

may be succinctly written as

$$
i_{\dot{\gamma}} d \sigma=0
$$

(see Ref. 3 and 4), where

$$
\dot{\gamma}=\dot{q}^{a} \partial_{q^{a}}+\dot{p}_{a} \partial_{p_{a}}+\partial_{t}
$$

is the velocity vector to curve $\gamma$ (on extended phase space) and

$$
\sigma=p_{a} d q^{a}-H d t
$$

is a distinguished 1-form, Poincaré-Cartan integral invariant (on extended phase space as well). Then, the standard action integral reads (see Refs. $\underline{3}$ )

$$
S[\gamma]=\int_{\gamma} \sigma=\int_{t_{1}}^{t_{2}}\left(p_{a} \dot{q}^{a}-H\right) d t
$$

Conserved quantities extracted from (continuous) symmetries of the action are functions on (extended) phase space (e.g. energy from time translations, components of momentum from space translations, components of angular momentum from rotations etc.). Explicitly (for details, including a derivation, see Appendix (A), if the symmetry is given by a vector field $\xi$ (on extended phase space), the corresponding conserved quantity $f_{\xi}$ is given as

$$
f_{\xi}=i_{\xi} \sigma-\chi_{\xi}
$$

In 1973 Nambu proposed a modification of Hamiltonian mechanics (Ref. 6). In its basic version, phase space is 3-dimensional (so that extended Nambu phase space is 4-dimensional) and equations of motion (Nambu equations) read

$$
\dot{x}_{i}=\epsilon_{i j k} \frac{\partial H_{1}}{\partial x_{j}} \frac{\partial H_{2}}{\partial x_{k}} \quad i=1,2,3
$$

or, in vector notation,

$$
\dot{\mathbf{r}}=\nabla H_{1} \times \nabla H_{2}
$$

Here, the two "Hamiltonians" are, in general, functions of $x_{1}, x_{2}, x_{3}$ and $t$.

It turns out that construction of action integral for Nambu mechanics is a slightly delicate subject. It was observed (Ref. 7 and 8) that Nambu equations (7) may be succinctly written as

$$
i_{\dot{\gamma}} d \hat{\sigma}=0
$$

too, where

$$
\dot{\gamma}=\dot{x}^{1} \partial_{1}+\dot{x}^{2} \partial_{2}+\dot{x}^{3} \partial_{3}+\partial_{t}
$$

is the velocity vector to curve $\gamma$ on extended Nambu phase space.

Equation (9) formally looks exactly like (2), it has the structure of "vortex-lines equation" (see Ref. 9). However, there is an important difference between the two equations, in that the form $\hat{\sigma}$, the counterpart of the one-form (4), is a two-form, now. Explicitly, it reads

$$
\hat{\sigma}:=x^{1} d x^{2} \wedge d x^{3}-H_{1} d H_{2} \wedge d t
$$

At first sight, the difference might look innocent. Notice, however, that it is no longer possible to write down action integral like (5), since there is no candidate for one-form to be integrated along the curve $\gamma$. Instead, the twoform $\hat{\sigma}$ is available. Therefore, the only way to produce a number (the value of action) is to integrate $\hat{\sigma}$ over a twodimensional surface.

In Ref. 7, a possibility to associate a surface with a single trajectory $\gamma$ is investigated. It leads to an action,

\footnotetext{
a) Electronic mail: fecko@fmph.uniba.sk
} 


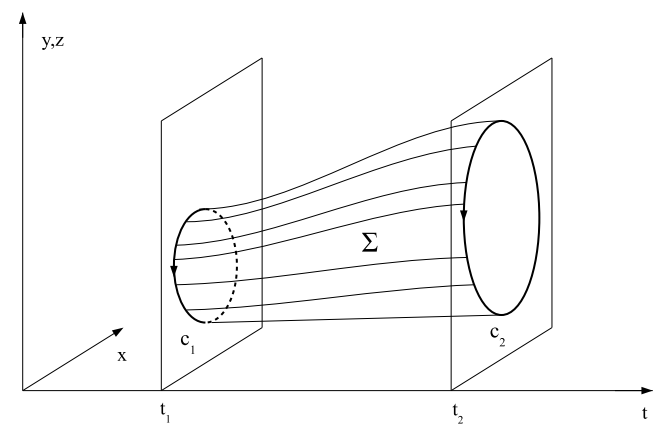

FIG. 1. A two-chain $\Sigma$ made up from a one-cycle $c_{1}$ using solutions of Nambu equations.

proposed already before in Ref. 10. This is not very satisfactory, since its extremals are curves on which $\dot{\mathbf{r}}$ is just proportional, not necessarily equal, to the r.h.s. of (8).

A more interesting way of how to come to a surface is proposed in Ref. 8. There, the value of action integral is associated with an appropriate one-parameter family of trajectories rather than with a single trajectory.

Namely, consider the family constructed as follows: Let, from each point $p$ of a one-cycle (loop) $c_{1}$ at the time $t_{1}$, emanate the solution $\gamma(t)$ of Nambu equations (9), fulfilling initial condition $\gamma\left(t_{1}\right)=p$. At the time $t_{2}$, the points $\gamma\left(t_{2}\right)$ (for all $p \in c_{1}$ ) form a one-cycle (loop) $c_{2}$ again (the image of $c_{1}$ w.r.t. the Nambu flow for $t_{2}-t_{1}$ ) and the points $\gamma(t)$, for all $t \in\left\langle t_{1}, t_{2}\right\rangle$ and all $p \in c_{1}$, form a two-chain (2-dimensional surface) $\Sigma$ made of solutions (see Fig 1 notice that $\partial \Sigma=c_{1}-c_{2}$ ). The value of the action, assigned to the family, is defined as

$$
S[\Sigma]=\int_{\Sigma} \hat{\sigma}
$$

One then easily verifies (see Appendix B) that the surface given by the family of solutions of Nambu equations is indeed an extremal of the action integral (12).

\section{SYMMETRIES OF THE ACTION}

Now, let us mimic in Nambu setting, i.e. using Takhtajan's action integral (12), the standard "Hamiltonian" procedure for obtaining conserved quantities from symmetries (see Appendix A).

So we call, first, vector field $\xi$ a symmetry if the action integral (12) evaluated on $\Phi_{\epsilon}(\Sigma)$ (the flow $\Phi_{\epsilon}$ corresponds to $\xi$, here) gives the same number as on $\Sigma$ itself

$$
S\left[\Phi_{\epsilon} \Sigma\right]=S[\Sigma]
$$

(i.e. $\delta S=0$; there are no restrictions on either time component of $\xi$ or the values of $\xi$ at the boundary $\partial \Sigma=$ $c_{1}-c_{2}$ of $\Sigma$ ). By direct computation of $\delta S$ (see Appendix (B), we obtain

$$
\delta S=\epsilon \int_{\Sigma} i_{\xi} d \hat{\sigma}+\epsilon \oint_{\partial \Sigma} i_{\xi} \hat{\sigma}
$$

Now, the first integral on the r.h.s. vanishes on the surface $\Sigma$ given by the family of solutions of Nambu equations (by the argument mentioned in Appendix $B ; \dot{\gamma}$ is tangent to $\Sigma$ and, at the same time, it is annihilated by $d \hat{\sigma})$. The second integral is over $\partial \Sigma=c_{1}-c_{2}$ and the sum of both integrals on the r.h.s. of (14) is to vanish. So we get

$$
0=\left(\oint_{c_{1}}-\oint_{c_{2}}\right) i_{\xi} \hat{\sigma}
$$

or, equivalently,

$$
\oint_{c_{1}} i_{\xi} \hat{\sigma}=\oint_{c_{2}} i_{\xi} \hat{\sigma}
$$

This is, however, nothing but a conservation law: for solutions of Nambu equations,

$$
f_{\xi}\left(t_{1} ; c_{1}\right)=f_{\xi}\left(t_{2} ; c_{2}\right)
$$

where $f_{\xi}$ is given by the integral

$$
f\left(t_{a} ; c_{a}\right):=\oint_{c_{a}} i_{\xi} \hat{\sigma} \quad a=1,2
$$

In full analogy with the Hamiltonian case (see the text following (A6) ), a more general definition of symmetry is possible. Rather than using differential version of (13), vanishing of the Lie derivative

$$
\mathcal{L}_{\xi} \hat{\sigma}=0
$$

we define symmetry of Nambu system as a vector field $\xi$ obeying somewhat weaker condition,

$$
\mathcal{L}_{\xi} \hat{\sigma}=d \chi_{\xi}
$$

(so, exactness of the Lie derivative is enough). Or, by Cartan's formula,

$$
i_{\xi} d \hat{\sigma}=-d\left(i_{\xi} \hat{\sigma}-\chi_{\xi}\right)
$$

Upon integration over the surface $\Sigma$ we get

$$
\int_{\Sigma} i_{\xi} d \hat{\sigma}=-\oint_{\partial \Sigma}\left(i_{\xi} \hat{\sigma}-\chi_{\xi}\right)
$$

Since the l.h.s. vanishes (on solutions), it holds

$$
\oint_{c_{1}}\left(i_{\xi} \hat{\sigma}-\chi_{\xi}\right)=\oint_{c_{2}}\left(i_{\xi} \hat{\sigma}-\chi_{\xi}\right)
$$

So, we obtain the statement

$$
f_{\xi}\left(t_{1} ; c_{1}\right)=f_{\xi}\left(t_{2} ; c_{2}\right)
$$

where $f_{\xi}$ is given by the integral

$$
f_{\xi}\left(t_{a} ; c_{a}\right):=\oint_{c_{a}}\left(i_{\xi} \hat{\sigma}-\chi_{\xi}\right) \quad a=1,2
$$


In more wordy formulation: Given a symmetry $\xi$ take, at time $t_{1}$, an arbitrary one-cycle (loop) $c_{1}$. Compute the line integral

$$
\int_{c_{1}}\left(i_{\xi} \hat{\sigma}-\chi_{\xi}\right)
$$

Then, let each point of $c_{1}$ evolve by Nambu flow up to time $t_{2}$. You get another one-cycle (loop), $c_{2}$. Compute, again, the line integral

$$
\int_{c_{2}}\left(i_{\xi} \hat{\sigma}-\chi_{\xi}\right)
$$

The statement is: You get the same number.

\section{INTEGRAL INVARIANTS}

What we obtained from symmetry $\xi$ is nothing but a relative integral invariant for Nambu dynamics. In general this is, by definition, a differential $p$-form $\alpha$ such that, when integrated over a $p$-cycle, it gives an invariant w.r.t. the dynamical flow. Put another way, if a dynamical vector field $\Gamma$ generates the flow $\Phi_{t}$ (time evolution) and if $c_{2}$ is the $\Phi_{t}$-image of an arbitrary $p$-cycle $c_{1}$, then,

$$
\oint_{c_{1}} \alpha=\oint_{c_{2}} \alpha
$$

(see, e.g., Refs. 3, , 5, 11, and 12).

In our case, the result (23) may be regarded as the statement that on Nambu extended phase space endowed with the dynamical vector field $\Gamma$ defined by

$$
i_{\Gamma} d \hat{\sigma}=0
$$

(see (9)) we get, as a consequence of existence of a symmetry $\xi$, a relative integral invariant. So (28) holds for the one-form

$$
\alpha=i_{\xi} \hat{\sigma}-\chi_{\xi}
$$

Of course, as is always the case, our relative integral invariant then automatically yields an absolute integral invariant, integral of the exterior derivative $d \alpha$ of $\alpha$ over any two-chain (two-dimensional surface) $s$. So, taking into account (21),

$$
\int_{s_{1}} i_{\xi} d \hat{\sigma}=\int_{s_{2}} i_{\xi} d \hat{\sigma}
$$

\section{AN APPROACH VIA MOMENTUM MAP}

Momentum map is a powerful tool for studying connection between symmetries and conserved quantities in Hamiltonian framework.

Standardly, it is a map from a symplectic manifold (phase space of a Hamiltonian system) to the dual of the
Lie algebra of the symmetry group acting on the phase space (see, e.g., Refs. 3, 4, and 13).

A slightly modified version enables one to treat momentum map as a map from extended phase space of the Hamiltonian system to the dual of the Lie algebra (see Appendix C).

Here we mimic the derivation of momentum map within the framework of the extended Nambu phase space.

So, consider an action of a Lie group $G$ on extended Nambu phase space. The action satisfies $R_{g}^{*} d \hat{\sigma}=d \hat{\sigma}$ or, infinitesimally, $\mathcal{L}_{\xi_{X}} d \hat{\sigma}=0$. This can be rewritten as closedness of two-form $\beta_{X}$

$$
d \beta_{X}=0 \quad \beta_{X}:=i_{\xi_{X}} d \hat{\sigma}
$$

(Notice that (32) is a statement concerning complete Nambu system, rather than just the extended Nambu phase space alone, since $\hat{\sigma}$ contains both "Hamiltonians" $H_{1}$ and $H_{2}$.) Often $\beta_{X}$ happens to be exact; then we get

$$
i_{\xi_{X}} d \hat{\sigma}=-d P_{X}
$$

Here, $P_{X}$ is a one-form (rather than a zero-form, i.e. a function, as in Hamiltonian case). We can achieve linearity of $P_{X}$ in $X$ in a standard way (see Ref. (4), write $P_{X}=X^{i} P_{i}$ and introduce

$$
P:=P_{i} E^{i}
$$

What we obtained is a $\mathcal{G}^{*}$-valued one-form on extended Nambu phase space (rather than the corresponding $\mathcal{G}^{*}$ valued zero-form $=$ function known from Hamiltonian framework as the momentum map). Its $j$-th component one-form, $P_{j}$, is defined, according to (33), by the equation

$$
i_{\xi_{E_{j}}} d \hat{\sigma}=-d P_{j}
$$

Comparison with (21) and (23) shows that in this way we get, as a reward for finding a symmetry, the same integral invariants as we found in Section [II.

$$
\oint_{c} P_{j}=\text { relative integral invariant }
$$

( $P_{j}$ equals $i_{\xi_{E_{j}}} \hat{\sigma}-\chi_{\xi_{E_{j}}}$ modulo additive closed one-form, vanishing after integration over the cycle) and in Section III,

$$
\int_{s} i_{\xi_{E_{j}}} d \hat{\sigma}=\text { absolute integral invariant }
$$

(see (31) and (35)).

So, to conclude, both approaches (the one discussed in Section 【 as well as the one discussed here) lead to the same picture regarding the relation between symmetries and conserved quantities due to them: each symmetry provides us with a relative integral invariant of the form (23) (and, consequently, with an absolute integral invariant of the form (31)). 
Let us remark that the question of momentum map in the context of Nambu mechanics was already addressed before in Ref. 14. There, it was introduced as a $\mathcal{G}^{*} \times \mathcal{G}^{*}$ valued mapping (i.e. function) defined by the formula

$$
i_{\xi_{X}} d \hat{\sigma}=d P_{1 X} \wedge d P_{2 X}
$$

where both components of the pair $\left(P_{1}, P_{2}\right)$ are defined by (C3), in which $M$ is Nambu phase space, now. Notice, however, that the formula (38) is inconsistent, since the l.h.s. is linear in $X$ whereas the r.h.s. is not.

\section{THE CASE OF MORE NAMBU HAMILTONIANS}

What we treated in detail was the "basic" version of Nambu mechanics, the situation, when the Nambu phase space is three-dimensional and there are two Nambu "Hamiltonians", $H_{1}$ and $H_{2}$.

Already in the original paper (Ref. 6) Nambu pointed out that the idea may be straightforwardly generalized to more dimensions, $n$-dimensional (Nambu) phase space and $n-1 \mathrm{Nambu}$ "Hamiltonians", $H_{1}, \ldots H_{n-1}$. (There are also other generalizations, see Refs. 6 and 8 .)

And it is easily seen that all constructions discussed in this paper work equally well in the $n$-dimensional version. In particular, $\hat{\sigma}$ becomes $(n-1)$-form, $c_{1}$ becomes $(n-2)$ cycle, $\Sigma$ is $(n-1)$-dimensional surface and so on. (See Refs. 8 and 9.) Conserved quantities are still integral invariants (formally equally looking formulas (23) and (31) hold, where $c_{a}$ are $(n-2)$-cycles and $s_{a}$ are $(n-1)$ chains).

\section{CONCLUSIONS}

Both Hamiltonian and Nambu mechanics study motion of (formally speaking) points in phase space (or, by technical reasons, in extended phase space). Therefore it is natural to expect conserved quantities to be functions on (perhaps extended) phase space. Once we study a particular motion, we evaluate the function at the time $t_{1}$ at the point where the motion begins, and then we profit from the fact that, at the future points of the trajectory, the same value of the function is guaranteed by the conservation law.

In Hamiltonian mechanics the story really goes like this. Functions (like energy or various components of momentum) are often conserved and this fact then makes life much more easy.

In Nambu mechanics, there are conserved functions as well. Already in the first paper on the subject (Ref. [6), Nambu discusses, as a key example, dynamical Euler equations for the motion of a free rigid rotator. Here, the Nambu phase space is three-dimensional (actually, it is just a subsystem of a complete six-dimensional Hamiltonian system of equations; one should add kinematical Euler equations to get the standard picture) and both energy and square of the angular momentum are conserved. Nambu shows that it is possible to choose these two (conserved) functions as the two "Hamiltonians" $H_{1}$ and $H_{2}$ in his approach. In many papers, thereafter, authors write various systems of ordinary differential equations in Nambu mechanics form, exactly to "make explicit" conserved quantities (functions, namely $H_{1}$ and $\mathrm{H}_{2}$ ).

However, the message of this paper is that these conserved functions do not directly follow from symmetries, as is usual in Hamiltonian case. In the case of symmetries, application of more or less standard machinery results, because of a peculiar situation with the action integral (presence of a two-form rather than one-form, necessity of taking a family of trajectories rather than a single trajectory), in conserved quantities, which have the character of integral invariants rather then usual conserved functions. Namely, the machinery leads to higherdegree forms rather than usual zero-forms, that is, functions (one-form for a relative invariant, and its exterior derivative, two-form, for the corresponding absolute invariant). As a reward for finding a symmetry, the conserved number is only obtained as integral of the form over a one-cycle (or two-chain for the absolute invariant).

In order to make the picture complete, let us note that also Liouville theorem holds in Nambu mechanics (phase volume is conserved) irrespective of concrete Nambu "Hamiltonians" (see Refs. 6 and 9). This means that there is an integral invariant available, not related to symmetries, too. (There is also whole series of wellknown Poincaré-Cartan integral invariants in Hamiltonian mechanics, with no relation to symmetries as well.)

So, there are altogether as many as three kinds of conserved quantities in Nambu mechanics. First, more common, evidently useful quantities (functions), which are, however, not related (at least in a clear way) to symmetries. Second, more exotic quantities (integral invariants), which, on contrary, result from application of standard machinery on symmetries. And third, the phase volume (integral invariant) which is not related to symmetries, again.

\section{Appendix A: Hamiltonian mechanics - extremals,} symmetries and conserved quantities

Here, in order to make comparison with Nambu mechanics easier, we recall briefly how standard reasoning goes in Hamiltonian mechanics. So, our action integral is given by (5).

On extended phase space, consider a vector field with vanishing time component (otherwise yet arbitrary), $W=W^{a} \partial_{q^{a}}+W_{a} \partial_{p_{a}}+0 . \partial_{t}$ ("variational" field). Its infinitesimal flow $\Phi_{\epsilon}$ performs ("equal time") variations of 
curves $\gamma \mapsto \gamma_{\epsilon}=\Phi_{\epsilon}(\gamma)$. Then $S[\gamma] \mapsto S\left[\gamma_{\epsilon}\right]$, where

$$
\begin{aligned}
S\left[\gamma_{\epsilon}\right] & =\int_{\gamma_{\epsilon}} \sigma=\int_{\gamma} \Phi_{\epsilon}^{*} \sigma=\int_{\gamma}\left(\hat{1}+\epsilon \mathcal{L}_{W}\right) \sigma \\
& =S[\gamma]+\epsilon \int_{\gamma} i_{W} d \sigma+\epsilon \int_{\gamma} d i_{W} \sigma \\
& =S[\gamma]+\epsilon \int_{\gamma} i_{W} d \sigma+\epsilon \int_{\partial \gamma} i_{W} \sigma
\end{aligned}
$$

So, we get for variation of action, $\delta S \equiv S\left[\gamma_{\epsilon}\right]-S[\gamma]$,

$$
\delta S=\epsilon \int_{t_{1}}^{t_{2}}\left\langle-i_{\dot{\gamma}} d \sigma, W\right\rangle d t+\left.\epsilon\left(p_{a} W^{a}\right)\right|_{t_{1}} ^{t_{2}}
$$

This means that, within the class of curves with fixed $q^{a}$ at $t_{1}$ and $t_{2}$ (this corresponds to $W^{a}\left(\gamma\left(t_{1}\right)\right)=0=$ $\left.W^{a}\left(\gamma\left(t_{2}\right)\right)\right)$, extremals of the action $(\delta S=0)$ coincide with solutions of equations of motion (2).

Now, vector field $\xi$ is a symmetry if the action integral (5) evaluated on $\Phi_{\epsilon} \circ \gamma$ (the flow $\Phi_{\epsilon}$ already corresponds to $\xi$, here) gives the same number as on $\gamma$ itself

$$
S\left[\Phi_{\epsilon} \circ \gamma\right]=S[\gamma]
$$

(i.e. $\delta S=0$; there are no restrictions on either time component of $\xi$ or the values of $\xi$ at the ends of $\gamma$ ). By the same direct computation as above, however, we obtain

$$
\delta S=\epsilon \int_{t_{1}}^{t_{2}}\left\langle-i_{\dot{\gamma}} d \sigma, \xi\right\rangle d t+\epsilon \int_{\partial \gamma} i_{\xi} \sigma
$$

Therefore, combining (A2), (A3) and (2) we see that on solutions of Hamilton equations one has

$$
0=\int_{\partial \gamma} i_{\xi} \sigma
$$

This is, however, nothing but a conservation law: on solutions of Hamilton equations,

$$
f_{\xi}\left(\gamma\left(t_{2}\right)\right)=f_{\xi}\left(\gamma\left(t_{1}\right)\right)
$$

for the function

$$
f_{\xi}:=i_{\xi} \sigma
$$

Actually, requiring (A2 is too restrictive. To see this notice that it is equivalent, according to the first line of the computation in the beginning of this section, to differential condition

$$
\mathcal{L}_{\xi} \sigma=0
$$

It turns out, however, that a more general definition of symmetry may be useful, namely as a vector field $\xi$ fulfilling just

$$
\mathcal{L}_{\xi} \sigma=d \chi_{\xi} \quad \text { i.e. } \quad i_{\xi} d \sigma=-d\left(i_{\xi} \sigma-\chi_{\xi}\right)
$$

(i.e. exactness of the Lie derivative is enough for gaining a conserved quantity, its vanishing being too strong requirement). Indeed, integrating (A8) over $\gamma$ gives

$$
\int_{t_{1}}^{t_{2}}\left\langle i_{\dot{\gamma}} d \sigma, \xi\right\rangle d t=\int_{\partial \gamma}\left(i_{\xi} \sigma-\chi_{\xi}\right)
$$

Since the l.h.s. vanishes (on solutions), we get conservation law (A5) for more general function, namely

$$
f_{\xi}:=i_{\xi} \sigma-\chi_{\xi}
$$

\section{Appendix B: Takhtajan's action and its extremals}

Let us proceed to the Nambu mechanics, now. Consider Takhtajan's action integral (12). Infinitesimal flow $\Phi_{\epsilon}$ of variational field $W=W^{x} \partial_{x}+W^{y} \partial_{y}+W^{z} \partial_{z}+0 . \partial_{t}$ performs ("equal time") variations of surfaces $\Sigma \mapsto \Sigma_{\epsilon}=$ $\Phi_{\epsilon}(\Sigma)$. Then $S[\Sigma] \mapsto S\left[\Sigma_{\epsilon}\right]$, where

$$
\begin{aligned}
S\left[\Sigma_{\epsilon}\right] & =\int_{\Sigma} \hat{\sigma}=\int_{\Sigma} \Phi_{\epsilon}^{*} \hat{\sigma}=\int_{\Sigma}\left(\hat{1}+\epsilon \mathcal{L}_{W}\right) \hat{\sigma} \\
& =S[\Sigma]+\epsilon \int_{\Sigma} i_{W} d \hat{\sigma}+\epsilon \int_{\Sigma} d i_{W} \hat{\sigma} \\
& =S[\Sigma]+\epsilon \int_{\Sigma} i_{W} d \hat{\sigma}+\epsilon \int_{\partial \Sigma} i_{W} \hat{\sigma}
\end{aligned}
$$

Now, on $\Sigma$ made of solutions of the equations of motion (9), integral $\int_{\Sigma} i_{W} d \hat{\sigma}$ vanishes, since we just sum terms proportional to $(d \hat{\sigma})(W, \dot{\gamma}, u)=-\left(i_{\dot{\gamma}} d \hat{\sigma}\right)(W, u)=0(u$ is tangent to the surface $\Sigma$, linearly independent of $\dot{\gamma}$; this is the counterpart of summing terms proportional to $(d \sigma)(W, \dot{\gamma})=\left(-i_{\dot{\gamma}} d \sigma\right)(W)=0$ in the Hamiltonian case $)$. So we get for variation of action, $\delta S \equiv S\left[\Sigma_{\epsilon}\right]-S[\Sigma]$, when computed on surface $\Sigma$ composed of solutions,

$$
\delta S=\epsilon\left(\oint_{c_{1}}-\oint_{c_{2}}\right) x\left(W^{y} d z-W^{z} d y\right)
$$

(since $i_{W} \hat{\sigma}=x\left(W^{y} d z-W^{z} d y\right)+(\ldots) d t$ and $d t$ vanishes on $c_{1}$ and $c_{2}$ ). This means that surfaces made of solutions provide extremals $(\delta S=0)$ in the class of surfaces whose boundaries, 1-chains $c_{1}$ at $t_{1}$ and $c_{2}$ at $t_{2}$ respectively, have fixed $y$ and $z$ values (put another way, fixed projections onto the $y z$-plane). This is because $W^{y}$ and $W^{z}$ should vanish at $t_{1}$ and $t_{2}$. (There is no need to fix $x$ at the ends. This is a counterpart of the Hamiltonian freedom to move $p_{a}$ at the ends.)

\section{Appendix C: Momentum map and extended phase space}

Most frequently, momentum map is associated with (certain) action of a Lie group $G$ on a phase space (symplectic manifold $(M, \omega)$; see Refs. 3, 4, and 13). The action should preserve the symplectic form, $R_{g}^{*} \omega=\omega$, so infinitesimally $\mathcal{L}_{\xi_{X}} \omega=0$ (where $\xi_{X}$ is the generator of the action, $X \in \mathcal{G})$. This can be rewritten as closedness of $\alpha_{X}$

$$
d \alpha_{X}=0 \quad \alpha_{X}:=i_{\xi_{X}} \omega
$$

Often $\alpha_{X}$ happens to be exact; then we get

$$
i_{\xi_{X}} \omega=-d P_{X} \quad P_{X}: M \rightarrow \mathbb{R}
$$

Finally, since linearity of $P_{X}$ w.r.t. $X$ may always be achieved, we can introduce momentum map as follows:

$$
P: M \rightarrow \mathcal{G}^{*} \quad\langle P(m), X\rangle:=P_{X}(m)
$$

Now, replace $\omega$ (on phase space) by $d \sigma$ (on extended phase space; $\sigma$ is given by (4))

$$
d \sigma=d p_{a} \wedge d q^{a}-d H \wedge d t
$$


So, consider an action of $G$ on extended phase space $M \times$ $\mathbb{R}$, such that $R_{g}^{*} d \sigma=d \sigma$, so infinitesimally $\mathcal{L}_{\xi_{X}} d \sigma=0$. This can be rewritten as closedness of $\beta_{X}$

$$
d \beta_{X}=0 \quad \beta_{X}:=i_{\xi_{X}} d \sigma
$$

(Notice that, unlike (C1), which says something about the phase space alone, with no reference to particular Hamiltonian governing the dynamics, (C5) is a statement concerning complete Hamiltonian system, since $\sigma$ contains $H$.) Often $\beta_{X}$ happens to be exact; then we get

$$
i_{\xi_{X}} d \sigma=-d P_{X} \quad P_{X}: M \times \mathbb{R} \rightarrow \mathbb{R}
$$

Finally, we can again introduce "momentum map" as follows:

$$
P: M \times \mathbb{R} \rightarrow \mathcal{G}^{*} \quad\langle P(m, t), X\rangle:=P_{X}(m, t)
$$

(Unlike (C2), we can not read (C6) as that the field $\xi_{X}$ is Hamiltonian; it lives on odd-dimensional manifold). This function (i.e. all component "ordinary" functions $P_{i}$, given by $\left.P=P_{i} E^{i}, P_{X}=X^{i} P_{i}\right)$ is conserved. Indeed, because of (C6) and (2) we can write

$$
\begin{aligned}
\dot{P}_{X} & \equiv \dot{\gamma} P_{X}=\left\langle d P_{X}, \dot{\gamma}\right\rangle=-(d \sigma)\left(\xi_{X}, \dot{\gamma}\right) \\
& =\left\langle i_{\dot{\gamma}} d \sigma, \xi_{X}\right\rangle=0
\end{aligned}
$$

so that

$$
\dot{P}_{i}=0 \quad i=1, \ldots, \operatorname{dim} \mathcal{G}
$$

Comparison of (A8) and (C6) reveals, that the two ways of obtaining conserved quantities from symmetry, discussed in Appendix A and C, respectively, yield the same result. (Modulo, of course, an additive constant function. One has to fix $X$ and call $\xi_{X} \equiv \xi$. Then $P_{X}$ from (C6) coincides with $f_{\xi}$ from (A10).)

${ }^{1}$ E. Nöther, Göttinger Nachrichten , 235-257 (1918).

${ }^{2} \mathrm{Y}$. Kosmann-Schwarzbach, The Nöther Theorems (Springer, 2011).

${ }^{3} \mathrm{~V}$. Arnold, Mathematical Methods of Classical Mechanics (Springer-Verlag, 1989).

${ }^{4}$ M. Fecko, Differential Geometry and Lie Groups for Physicists (Cambridge University Press, 2006).

${ }^{5}$ L. D. Landau and E. M. Lifshitz, Mechanics, 3-rd ed. (Butterworth-Heinemann Ltd, 1995).

${ }^{6}$ Y. Nambu, Phys. Rev. D 7, 2405-2412 (1973).

${ }^{7}$ M. Fecko, J. Math. Phys. 33, 930-933 (1992).

${ }^{8}$ L. Takhtajan, Comm. Math. Phys. 160, 295-315 (1994), hep-th/9301111

${ }^{9}$ M. Fecko, (2013), math-ph/1305.3167v1

${ }^{10}$ F. Bayen and M. Flato, Phys. Rev. D 11, 3049-3053 (1975).

${ }^{11}$ E. Cartan, Lecons sur les invariants intégraux (1922).

${ }^{12}$ L. Pars, A Treatise on Analytical Dynamics (Heinemann, 1965).

${ }^{13}$ M. Crampin and F. A. E. Pirani, Applicable Differential Geometry (Cambridge University Press, 1986).

${ }^{14}$ S. A. Pandit and A. D. Gangal, (1999), math/9908023v1 